\title{
Clinical, Ultrasound, and Predictability Outcomes Following Certolizumab Pegol Treatment (with Methotrexate) in Patients with Moderate-to-Severe Rheumatoid Arthritis: 52-Week Results from the CZP- SPEED Study
}

Piercarlo Sarzi-Puttini · Emilio Filippucci - Silvano Adami · Pier Luigi Meroni · Alberto Batticciotto •

Luca Idolazzi · Orazio De Lucia · Pablo Talavera · Thomas Kumke • Walter Grassi

Received: May 10, 2018 / Published online: July 24, 2018

(C) The Author(s) 2018

\section{ABSTRACT}

Introduction: To assess the impact of certolizumab pegol (CZP) treatment on clinical, patient-reported, and musculoskeletal ultrasound outcomes and to determine the treatment response time point most predictive of long-term outcomes in Italian patients with rheumatoid arthritis (RA).

Methods: CZP-SPEED (NCT01443364) was a 52-week, open-label, prospective, interventional, multicenter study. Biologic-naïve patients with moderate-to-severe active RA,

Prof. Adami sadly passed away during the development of this publication.

Enhanced Digital Features To view enhanced digital features for this article go to https://doi.org/10.6084/ m9.figshare.6736469.

Electronic supplementary material The online version of this article (https://doi.org/10.1007/s12325018-0751-8) contains supplementary material, which is available to authorized users.

P. Sarzi-Puttini · A. Batticciotto

Azienda Ospedaliera Luigi Sacco Polo Universitario, Milan, Italy

E. Filippucci · W. Grassi ( $₫)$

Università Politecnica delle Marche, Ancona, Italy

e-mail: walter.grassi@univpm.it

S. Adami · L. Idolazzi

Università degli Studi di Verona, Azienda

Ospedaliera di Verona, Verona, Italy who had failed at least one DMARD treatment, received CZP (400 $\mathrm{mg}$ at weeks 0,2 , and 4 , then $200 \mathrm{mg}$ every 2 weeks) concomitantly with methotrexate. The primary objective was to identify the time point of clinical response \{decrease in 28-joint Disease Activity Score $[$ DAS28(ESR)] $\geq 1.2\}$ most predictive of a clinical response at week 52. Additional clinical and patient-reported outcomes were measured. Power Doppler (PD) ultrasound was used to assess synovial effusion, synovial proliferation, PD signal, cartilage damage, and bone erosion according to international guidelines.

Results: A total of 132 patients were enrolled and received CZP; 91/132 (69\%) completed to week 52. Predicted 52-week responses for early responders (week 2 onwards) were between $65 \%$ and $70 \%$. Rapid improvements in joint cavity widening and PD signal were observed to week 8 and maintained to week 52. Cartilage damage and bone erosion were stable over 52 weeks. No new safety signals were identified.

Conclusion: In Italian CZP-treated patients with moderate-to-severe RA, week 12 clinical

\section{P. L. Meroni · O. De Lucia}

ASST Centro Ortopedico Traumatologico Gaetano Pini-CTO-Università degli Studi di Milano, Milan, Italy

P. Talavera

UCB Pharma, Madrid, Spain

T. Kumke

UCB Pharma, Monheim, Germany 
responses may be predictive of long-term response at week 52. Rapid improvements in clinical, patient-reported, and musculoskeletal ultrasound outcomes were maintained to week 52. These data may aid rheumatologists to make earlier treatment decisions.

Trial Registration: ClinicalTrials.gov identifier, NCT01443364.

Funding: UCB Pharma.

Keywords: Anti-TNF; Certolizumab pegol; Patient-reported outcome; Predictability; Rheumatoid arthritis; Rheumatology;

Ultrasound

\section{INTRODUCTION}

Tumor necrosis factor alpha inhibitors (antiTNFs) are an established treatment option for rheumatoid arthritis (RA), capable of ameliorating disease activity, slowing progression of joint damage, and improving health-related quality of life [1].

Certolizumab pegol (CZP) is a PEGylated, Fcfree anti-TNF that, owing to its innovative structure, exerts a prolonged half-life [2] and potentially different pharmacokinetic properties compared to other anti-TNF agents [3]; evidence from murine models suggests that CZP may be able to penetrate inflamed arthritic tissue to a greater extent compared to infliximab and adalimumab [3-6].

Previous research from the RAPID-1 trial indicates that patients who do not achieve a 28-joint Disease Activity Score [DAS28(ESR)] response within the first 12 weeks of CZP and methotrexate (MTX) combination therapy are unlikely to reach low disease activity (LDA) at 12 or 52 weeks $[7,8]$. However, since these observations were based on post hoc analyses, further studies are required to support these predictability results. This early identification of patients who do not respond to CZP therapy can help physicians follow treat-to-target recommendations [9], facilitating earlier reassessment of current treatment. This is likely to lead to more rapid achievement of clinical goals.

Power Doppler ultrasound (PDUS) is a straightforward, noninvasive imaging instrument employed to evaluate both clinical and subclinical synovitis [10-15], which can be used alongside clinical and patient-reported outcomes (PROs) in order to assess disease activity. Prior research on the usage of PDUS in rheumatology has proven its reliability and sensitivity. This tool is able to visualize the synovial inflammatory joint changes in RA that may have previously gone undetected by conventional clinical and radiographic examinations [10]. Furthermore, previous studies suggest that PDUS brings additional value alongside clinical examination, both in improving early diagnosis of RA through the detection of subclinical synovitis as well as establishing whether true RA remission has been achieved [16-18]. Thus PDUS can be used to support the diagnosis process and may help to predict long-term clinical outcomes-an indispensable advantage for clinicians. According to the Italian Society of Rheumatology (SIR) recommendations for the use of biologics in RA, synovitis assessed by PDUS may be considered as an image biomarker for poor prognostic features $[17,19]$.

The primary objective of this study was to identify the time point of clinical response with the highest predictive value at week 52 . The secondary study objective was to assess the PDUS response over time in a subgroup of patients. In order to achieve these objectives, CZP efficacy, safety, and predictability in terms of clinical, PROs, and PDUS outcomes were assessed over 52 weeks in a cohort of Italian patients with moderate-to-severe RA. We hypothesized that CZP treatment would improve clinical outcomes, PROs, and PDUS outcomes after 52 weeks.

\section{METHODS}

\section{Study Design}

The CZP-SPEED study (NCT01443364 and EudraCT 2011-000385-35) was a 52-week, openlabel, prospective, interventional, multicenter study which evaluated the predictability of disease control at week 52 based on early response to CZP (in combination with MTX) in Italian patients with RA. CZP was supplied by UCB Pharma. Concomitant use of oral corticosteroids 
( $\leq 10 \mathrm{mg} /$ day prednisone, or equivalent) and non-steroidal anti-inflammatory drugs (NSAIDs) was permitted at stable doses during the course of the study. Analgesics without anti-inflammatory action and oral opioid analgesics were also permitted during the study but could not be used prior to clinical assessments on the day of a scheduled visit. Assessments were carried out at weeks $0,1,2,4,6,8,12,24,36$, and 52 .

\section{Patients}

Patients enrolled in the trial were biologicnaïve, had moderate-to-severe RA (defined as at least six swollen and at least six tender joints) and had failed treatment with at least one disease-modifying anti-rheumatic drug (DMARD); which is in line with the indication of CZP stated in the approved label [Summary of product Characteristics (SmPC)] [20]. Patients were treated with CZP (400 mg at weeks 0, 2 and 4, followed by $200 \mathrm{mg}$ every 2 weeks) in combination with MTX ( $\geq 15$ or $\leq 25 \mathrm{mg}$ per week, unless limited by subject intolerance or toxicity). The doses of CZP used in the study were in agreement with the approved label (SmPC) for treatment of RA with CZP [20]. The safety set (SS) consisted of all patients who received at least one dose of CZP. The full analysis set (FAS) consisted of all patients in the SS who had a valid baseline and post-baseline efficacy measurement for the primary objective. A subgroup of these patients (the PDUS subgroup) provided additional ultrasound and radiographic imaging evaluations if they had been diagnosed with RA for at most 2 years and they presented with at least two of the following PDUS findings: synovial proliferation, synovial vascularization, or presence of fluid in at least one metacarpophalangeal (MCP) joint.

\section{Compliance with Ethics Guidelines}

All procedures performed in studies involving human participants were in accordance with the ethical standards of the responsible Italian authorities [the Agenzia Italiana del Farmaco (AIFA)] and with the 1964 Helsinki declaration and its later amendments or comparable ethical standards. Informed consent was obtained from all participants included in the study.

\section{Study Procedures and Evaluations}

The primary objective was to detect the time point of clinical response with the highest positive predictive value (PPV). PPV was defined as the percentage of patients with a clinical response [reduction from baseline in DAS28(ESR) of at least 1.2] up to week 12, who also had clinical response [reduction from baseline in DAS28(ESR) of at least 1.2] at week 52.

Additional clinical exploratory variables included American College of Rheumatology ACR20/ 50/70 response [21], DAS28(ESR), DAS28(CRP), Patient Global Assessment of Disease Activity (PtGADA) [22], Physician Global Assessment of Disease Activity (PhGADA), Patient Assessment of Arthritis Pain (PtAAP), and Health Assessment Questionnaire Disability Index (HAQ-DI) [21].

\section{Imaging Evaluations}

The secondary objective of the study was to observe the effectiveness of PDUS in assessing both disease activity and impact of treatment in the subgroup of subjects undergoing PDUS. Additionally, the degrees of joint damage of the hands, wrists, and feet were assessed radiographically using the van der Heijde modified total Sharp score (mTSS) in the PDUS subgroup of patients $[23,24]$.

The second and third MCP joints, bilaterally together with another MCP joint (the most clinically involved), were examined by ultrasound measurements at weeks $0,1,2,4,6,8,12$, 24, 36, and 52. Ultrasound variables under evaluation were joint cavity widening, synovial proliferation, Power Doppler (PD) signal (synovial vascularization), bone erosion, and cartilage damage score measurements according to international guidelines. Joint cavity widening and PD signal were scored from 0 to 3 depending on the extent of synovial effusion and/or proliferation $[25,26]$ and the level of PD signal at the synovial tissue [27, 28], respectively. Cartilage damage and bone erosions were scored from 0 to 4 on the basis of metacarpal cartilage thickness at the MCP joint level 
$[29,30]$ and diameter of bone erosion per joint $[26,31]$, respectively. PDUS sum scores were the sum of the scores generated using these two semiquantitative scoring systems [25, 27].

A standardized instrumentation (MyLab ${ }^{\mathrm{TM}} 70$ XVG or Mylab ${ }^{\mathrm{TM}}$ Twice-Esaote Biomedica, or GE Logiq 9, equipped with a broadband linear probe of $6-18 \mathrm{MHz}$ ) and operational technique was adopted with multiplanar scanning technique on dorsal, lateral, and volar aspects of the MCP joints. All sonographers were trained before the start of the study. All images were reassessed by an assigned ultrasound expert reader and analyzed for inter-reader reliability.

\section{Safety Measurements}

Safety measurements assessed included adverse events (AEs) classified by system organ class (SOC) and preferred term (PT) using the Medical Dictionary for Regulatory Activities (MedDRA) version 18.0. Only treatment-emergent AEs (TEAEs) were included in the summary tables.

\section{Statistical Analysis}

The SS consisted of all enrolled patients who received at least one dose of study medication. Clinical outcomes and PROs were assessed in the full analysis set (FAS), defined as all patients who received at least one dose of CZP and had at least one baseline and at least one postbaseline DAS28(ESR) assessment (the primary clinical variable). PDUS outcomes were assessed in a subgroup of enrolled patients with RA disease duration less than 2 years and proof of at least two of the following: synovial proliferation, synovial vascularization, and presence of fluid in at least one selected joint.

A sample size of 120 patients was calculated to be appropriate to produce $95 \%$ confidence intervals (CIs) with sufficient precision $( \pm 0.069)$ to estimate predictability for the base case of a $60 \%$ response rate. Precision would be higher if the proportion of responders was greater than $60 \%$. At least $40(33.3 \%)$ of the 120 included patients were planned to be part of the PDUS subgroup of patients.

In order to analyze the primary objective, DAS28(ESR) was measured at weeks 1, 2, 4, 6, 8, and 12 and was used to predict maintenance of long-term efficacy in CZP-treated patients, using the PPV and negative predictive values (NPV). Logistic regression was performed post hoc to evaluate the predictive ability of early responders, up to week 12, and long-term efficacy at week 52, after controlling for the prognostic factors [baseline DAS28(ESR), gender, age, and duration of RA]. Area under curve (AUC) was obtained from fitting the logistic regression model with week 52 response as the dependent variable and early response (weeks 1-12) as the independent variable. The model with the maximum AUC was considered the better predictor.

PPV was defined using the equation: [32] $\frac{n}{N} \times$ 100 , where $n$ is the number of patients with a clinical response at week 52 and a clinical response at a specified early time point, and $N$ is all patients with a clinical response at a specified early time point. Clinical response was defined as a reduction in DAS28(ESR) of at least 1.2. For $\mathrm{NPV}, n$ is the number of patients who did not have a clinical response at week 52 nor a clinical response at a specified early time point.

A mixed effects linear model for repeated measures was used to examine the effect of each of the prognostic factors on the sum scores describing disease activity (joint cavity widening, synovial proliferation, and PD signal/blood flow) and disease severity (progression in cartilage damage and bone erosions). Within this model, "week" was a repeated measure and the prognostic factors [baseline DAS28(ESR), gender, age, duration of RA] were fixed effects. The regression coefficient, standard error (SE), and $p$ values associated with each prognostic factor are presented. During a post hoc analysis, patient duration of RA and the presence or absence of bone erosion were assessed for PDUS outcomes. Correlation analysis was conducted between PDUS and mTSS at each visit using the Spearman's correlation coefficient. Inter-reader reliability of all six joints was assessed in a subgroup of the PDUS patients at baseline and weeks 6,12 , and 36 using a blinded expert to review the images for PD signal/blood flow and cartilage damage. The first and second readings of the images were analyzed for inter-reader reliability using Cohen's kappa statistics. 


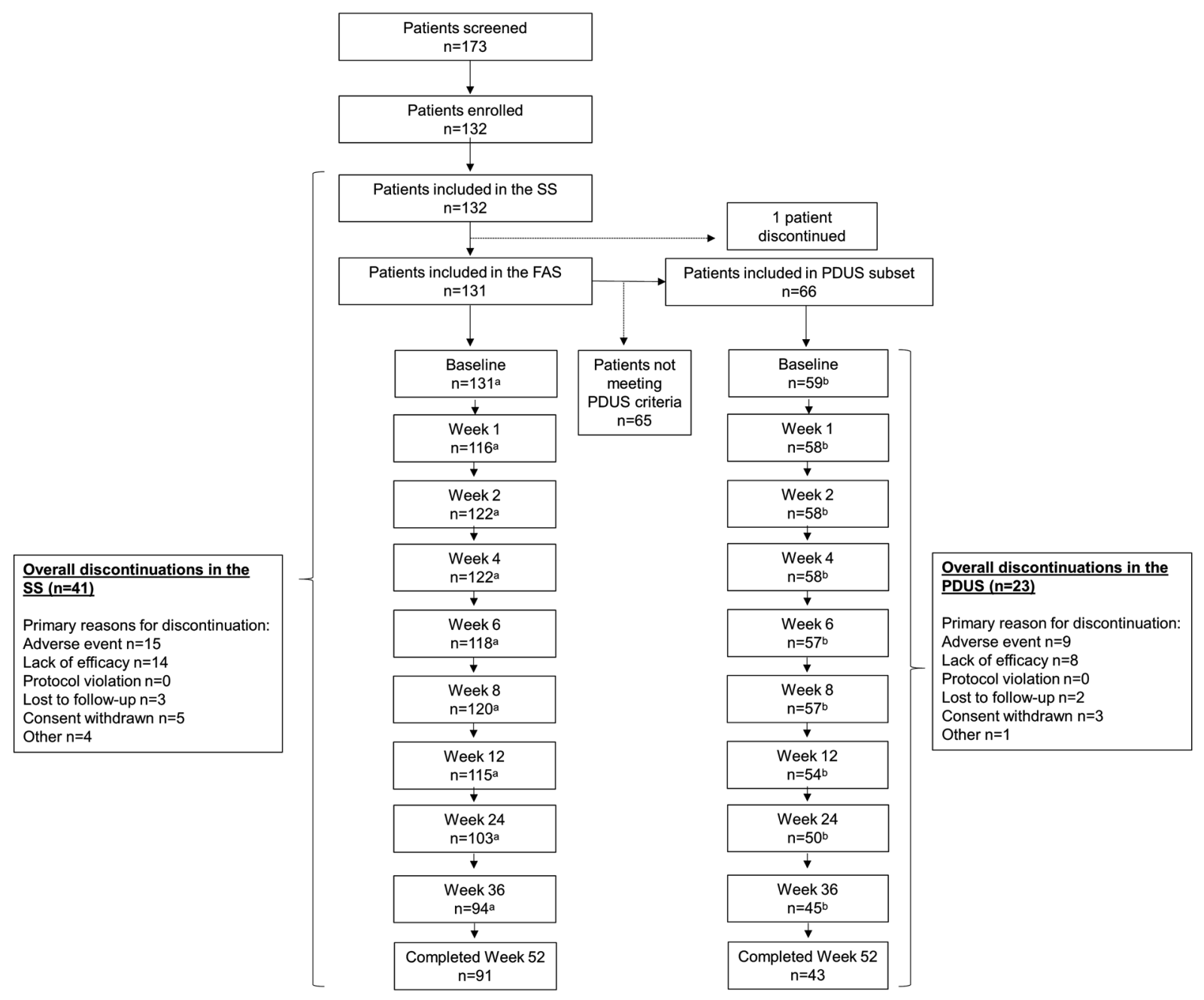

Fig. 1 CONSORT diagram showing patient disposition throughout the SPEED study. ${ }^{a}$ Patients who provided DAS28(ESR) values (observed case). ${ }^{\mathrm{b}}$ Patients who provided joint cavity widening and synovial proliferation

values (observed case). FAS full analysis set, PDUS Power Doppler ultrasound, SS safety set

Missing data were imputed using non-responder imputation (NRI) for categorical data and last observation carried forward (LOCF) for continuous data.

\section{RESULTS}

\section{Patient Disposition and Baseline Characteristics}

In total, 132 patients were enrolled and received CZP (SS) between December 2011 and May 2015, of whom 131 (99\%) comprised the FAS,

and 91 (69\%) completed to week 52 (Fig. 1). The primary reasons for discontinuation included adverse events (15 patients), lack of efficacy (14 patients), loss to follow-up (3 patients), consent withdrawn (5 patients), and other (4 patients) (Fig. 1). The PDUS subgroup consisted of 66 (50.0\%) patients, and 43/66 (65.2\%) PDUS patients completed to week 52 (Fig. 1). The primary reasons for discontinuation in the PDUS subgroup included adverse events (9 patients), lack of efficacy (8 patients), loss to follow-up ( 2 patients), consent withdrawn (3 patients), and other (1 patient) (Fig. 1). 
Table 1 Patient characteristics at baseline for the full analysis set and PDUS subgroup

\begin{tabular}{llc}
\hline Mean (SD), unless otherwise stated & Full analysis set $\boldsymbol{n}=\mathbf{1 3 1}$ & PDUS subgroup $\mathbf{n}^{\mathbf{n}=\mathbf{6 6}}$ \\
\hline Age, years & $54.8(13.2)$ & $53.4(13.7)$ \\
Female, $n$ (\%) & $107(81.7)$ & $54(81.8)$ \\
Disease duration, median (min; max) & $1.8(0 ; 5)$ & $1.5(1 ; 2)$ \\
$\leq 2$ years, $n$ (\%) & $88(67.2)$ & $66(100.0)$ \\
$>2$ years, $n$ (\%) & $43(32.8)$ & $0(0.0)$ \\
With extra-articular features, $n$ (\%) & $16(12.2)$ & $7(10.6)$ \\
Duration of morning stiffness, median hours (min; max) & $1.0(0 ; 24)$ & $1.0(1 ; 24)$ \\
$<2$ h, $n$ (\%) & $92(70.2)$ & $51(77.3)$ \\
$\geq 2$ h, $n$ (\%) & $39(29.8)$ & $15(22.7)$ \\
DAS28(ESR) & $5.9(1.1)$ & $5.7(1.0)$ \\
DAS28(CRP) & $5.3(1.0)$ & $5.1(1.0)$ \\
HAQ-DI & $1.3(0.7)$ & $1.3(0.7)$ \\
PtAAP & $56.5(23.4)$ & $53.8(24.3)$ \\
PtGADA & $57.3(23.2)$ & $53.5(24.1)$ \\
PhGADA & $56.6(19.1)$ & $52.0(18.9)$ \\
PDUS sum score & - & $20.5(11.7)$ \\
mTSS, median (min; max) & $20.0(0 ; 121)$ & $20.0(0 ; 121)$ \\
ESR, median mm/h (min; max) & $35.0(2 ; 140)$ & $32.0(2 ; 105)$ \\
CRP median mg/L (min; max) & $6.4(0 ; 201)$ & $5.3(0 ; 201)$ \\
Bone erosion, $n$ (\%) & $45(34.4)$ & $43(65.2)$ \\
\hline & & \\
\hline
\end{tabular}

CRP C-reactive protein, DAS28 28-joint disease activity score, ESR erythrocyte sedimentation rate, FAS full analysis set, HAQ-DI health assessment questionnaire-disability index, $m$ TSS modified total Sharp score, PDUS Power Doppler ultrasound score, PtAAP patient's assessment of arthritis pain, PtGADA patient's global assessment of disease activity, $P h G A D A$ physician's global assessment of disease activity, $V A S$ visual analogue scale

${ }^{a}$ PDUS subgroup defined as patients with duration of RA $<2$ years

$\mathrm{b}$ Bone erosion measured by ultrasound and reported as number of erosions

Baseline characteristics were similar between the FAS and PDUS subgroup (Table 1). Characteristics by duration of RA prior to study enrollment are shown in Supplementary Table S1. At baseline, 131/132 (99.2\%) enrolled patients had previously taken, or were taking, concomitant DMARDs, including methotrexate [126 (95.5\%) patients], methotrexate sodium [6 (4.5\%) patients], and leflunomide [3 (2.3\%) patients]. Prior and concomitant use of permitted medications (corticosteroids, NSAIDs, non-antiinflammatory analgesics, and oral opioid analgesics) are reported in Supplementary Table S2.

\section{Predictability}

DAS28(ESR) response [DAS28(ESR) $\geq 1.2$ from baseline] rates were $70 \%$ by week 4 of treatment and remained at a similar level for the 


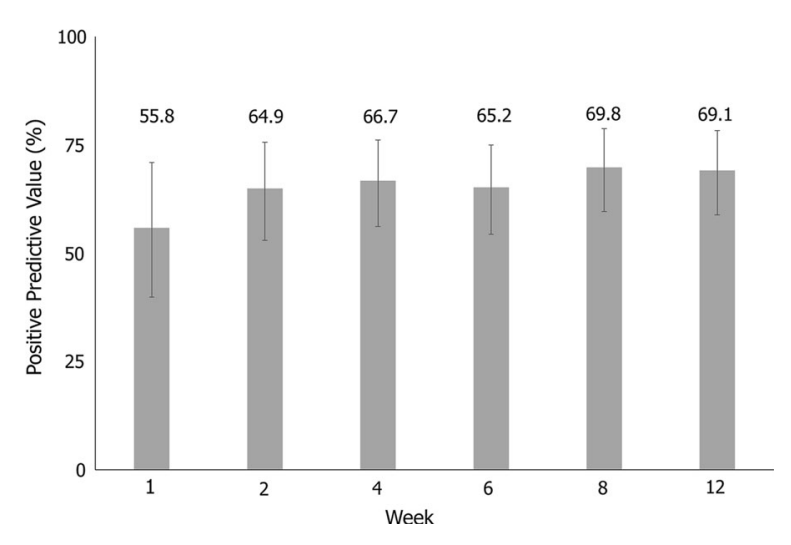

Fig. 2 Positive predictive value (PPV): percentage of patients with DAS28(ESR) clinical response at week 52 who also had clinical response at an early time point. Data reported for the full analysis set, $n=131$. Missing values were imputed using non-responder imputation (NRI). Clinical response was defined as a reduction of at least 1.2 in DAS28(ESR). DAS28 28-joint disease activity score, ESR erythrocyte sedimentation rate, $N R I$ non-responder imputation, $P P V$ positive predictive value

remainder of the trial (Supplementary Fig. S1). Patients with disease duration of at most 1 year were less likely to experience DAS28(ESR) response compared to patients with more established disease (Supplementary Fig. S2b). Of the patients who responded with a DAS28(ESR) reduction of at least 1.2 at an early time point (week 2-12), this clinical response was still observed for $65-70 \%$ at week 52 (Fig. 2).

The week with the numerically greatest PPV was week 8 , with $69.8 \%$ responders reporting at least a 1.2 point reduction in DAS28(ESR) at week 52; however, the predictive values of all time points from week 2 onwards were comparable (Fig. 2). Patients with disease duration of over 1 year had higher PPV and lower NPV outcomes compared to patients with shorter disease duration at all time points (Fig. 3). The post hoc logistic regression analysis did not identify any significant prognostic factors for predictability (Fig. 4).

\section{Clinical and Radiographic Outcomes}

Rapid improvements in clinical and patient-reported outcomes were observed from week 1 to week 12 and were maintained to week 52 (Fig. 5), including an improvement in DAS28(ESR) of -2.31 from baseline to week 52 (Fig. 5a). However, no significant correlation between PDUS outcomes and DAS28(ESR) was observed.

The proportion of patients achieving ACR20/ $50 / 70$ increased to week $12(67.2 \% / 41.2 \% /$ $23.7 \%)$ and was maintained to week 52 (61.8\%/ 47.3\%/29.8\%) (Fig. 5b). Mean improvement in HAQ-DI of -0.57 from baseline to week 52 (Fig. 5c) and in PtAAP (- 26.2 change from baseline to week 52; Fig. 5d) was observed. Within the PDUS subgroup, mTSS scores remained approximately constant over the course of the study [median (standard deviation; SD) 20.0 (32.0) at baseline, median change from baseline 1.0 (15.8) at week 52] suggesting inhibition of radiographic progression in these patients (Supplementary Fig. S5).

\section{PDUS Outcomes}

Rapid reductions in joint cavity widening were observed to week 8 across joints analyzed, which were maintained to week 52 (Fig. 6a). Patients with presence of bone erosion had higher levels of joint cavity widening at baseline and experienced the greatest levels of joint cavity widening reduction over time (Supplementary Figs. S3a, b). There was also a rapid decrease in the PD signal at a joint level by week 8 , which was maintained through week 52 (Fig. 6b). Patients with measurable bone erosion at baseline had higher PD signals overall, but also reported a greater level of improvement following CZP treatment (Supplementary Figs. S3c, d). Cartilage damage and bone erosion were stable over 52 weeks (Supplementary Fig. S4), indicating a lack of progression during the treatment period. Similarly, radiographic analysis of joints by mTSS showed that joint damage remained stable over 52 weeks (Supplementary Fig. S5). Correlation analysis at weeks 4,12 , and 52 between PDUS and mTSS resulted in correlation coefficients of at least 0.526 and $p<0.001$. Inter-reader reliability of the readings of PDUS images (representative PDUS images provided in Supplementary 
(a)

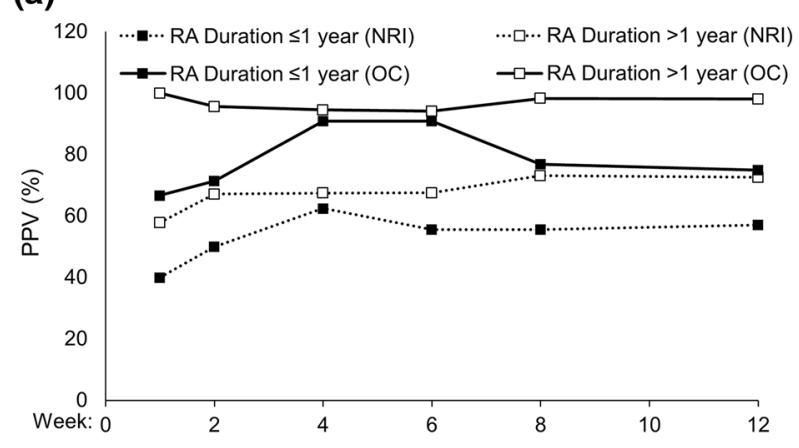

(c)

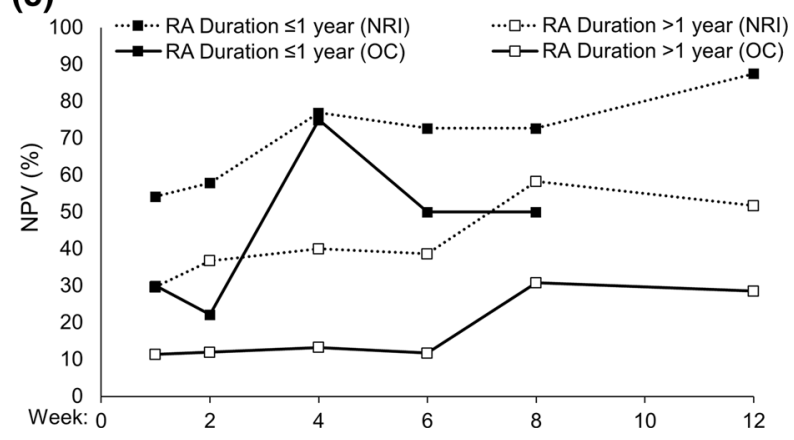

Fig. 3 Evaluation of a positive predictive values (PPV), $\mathbf{b}$ area under the curve (AUC), and $\mathbf{c}$ negative predictive values (NPV) of clinical response at time points up to week 12. Data are reported for the full analysis set (RA duration of at most 1 year, $n=29$; RA duration greater than 1 year, $n=102)$. Missing data were imputed using non-responder imputation (NRI). Data are provided for observed cases (OC) and NRI. Clinical responses at week 1 to week 12

Fig. S6) ranged from good (kappa $>0.75$ ) at week 0 to moderate (kappa 0.4-0.75) at later time points in the study.

The mixed effect with repeated measures analysis indicated that duration of RA $(<1$ year vs. $\geq 1-<2$ years) had a significant effect on the sum of the joint cavity widening, synovial proliferation, and PD signal (Table 2). Similar results were reported for the sum of the progression of cartilage damage and bone erosion scores (Table 2). Study site was not included as a fixed effect in these analyses as patient enrollment by study center was low (at most 11 patients). Two study sites had higher patient enrollment (24 and 27 patients) and the inclusion of site as a fixed variable (sites with high enrollment vs others) showed that the former did not have a significant effect on clinical (b)

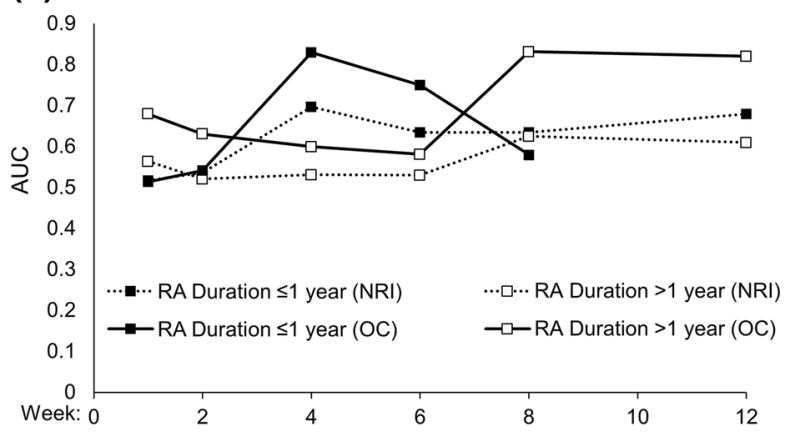

were defined as a reduction of at least 1.2 from baseline in DAS28(ESR). AUC and NPV data were unavailable for patients with RA duration of at most 1 year (OC) at week 12. $A U C$ area under the curve, $N P V$ negative predictive value, $N R I$ non-responder imputation, $O C$ observed case, $P P V$ positive predictive value, $R A$ rheumatoid arthritis

response at week 52 ( $p>0.05$ for both sites). A post hoc analysis also suggested that reductions in joint cavity widening were greatest and PD signals were lowest in patients with RA disease duration of 1 year or less (Fig. 6c, d).

\section{Safety}

During the trial, 301 AEs occurred in 94 patients (71.2\%) and 25 of these AEs were considered serious (Supplementary Table S3). The most common TEAEs occurring during the study were within the MedDRA SOC "infections and infestations", which occurred in 49 patients (37.1\%), including influenza viral infections (15 patients, $11.4 \%$ ), herpes viral infections (12 patients, 9.1\%), and upper and lower respiratory 


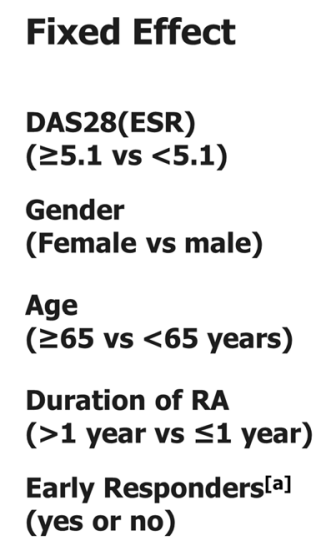

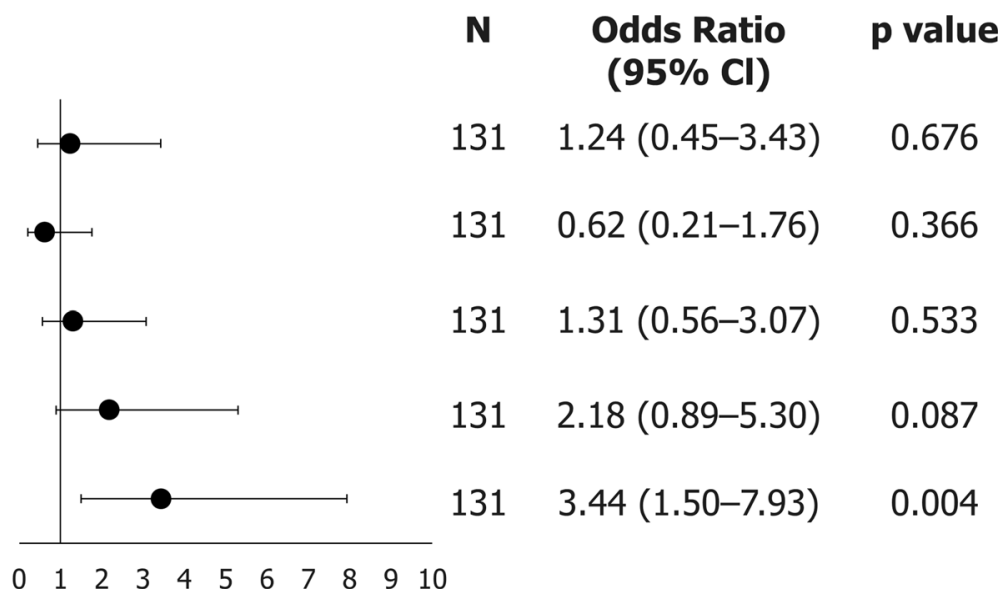

Odds Ratio p value \% Cl) effects and baseline DAS28(ESR) score, gender, age, and duration of RA as factors. An odds ratio greater than 1 indicates a higher response rate in the first category [e.g., DAS28(ESR) $\geq 5.1]$ compared to the second category [e.g., DAS28(ESR) < 5.1]. CI confidence interval, DAS28 28-joint disease activity score, ESR erythrocyte sedimentation rate, $R A$ rheumatoid arthritis

factors may not have been sufficient to identify changes; additionally, other baseline characteristics that were not selected and tested might have had an impact on predicting long-term response to CZP. The DAS28(ESR) results demonstrate that there was a "predictive window", between week 4 and week 12, which was the most predictive of a long-term response to treatment. Patients with RA disease duration of 1 year or less had numerically lower rates of DAS28(ESR) response compared to patients with more established disease. Similarly, patients with disease duration of at least 1 year had higher PPV outcomes compared to patients with shorter disease duration. This does not align with previous reports in which disease duration was not a significant predictor of good DAS28 response rates or ACR20 response rates $[33,34]$. The improvements in patient-reported HAQ-DI and PtAAP from baseline to week 52 show that in addition to the observed improvements in disease activity, patients experienced a better health-related quality of life, with less pain, over the duration of the study. The proportion of patients in the FAS completing the study to week 52 was $70 \%$ 
(a) DAS28(ESR) [LOCF]

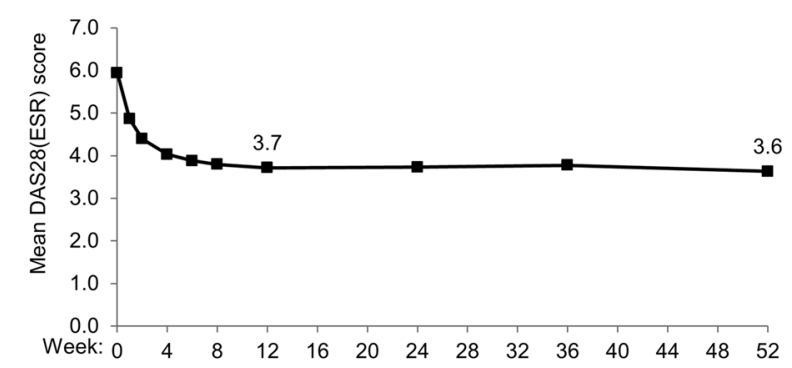

(c) HAQ-DI [LOCF]

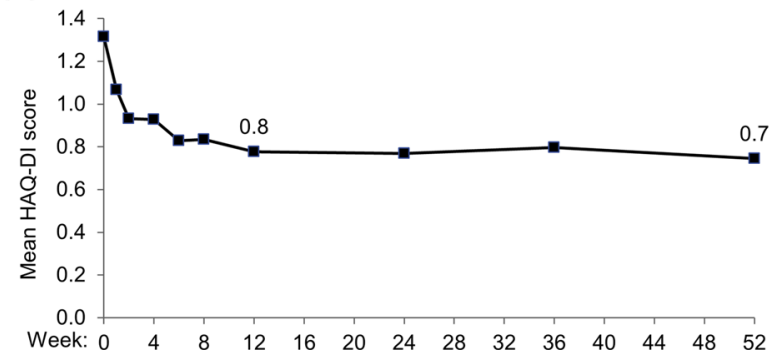

Fig. 5 Improvements in clinical and patient-reported outcomes up to week 52 of the CZP-SPEED study. Data are reported for the full analysis set $(n=131)$. Missing data were imputed using last observation carried forward for continuous variables and non-responder imputation for binary data. Patients rated their level of arthritis pain on a $100 \mathrm{~mm}$ VAS, where 0 indicated "no pain" and 100 indicated "most severe pain". DAS28 28-joint disease

which is in line with the retention rates observed in the RAPID-1 and RAPID-2 randomized clinical trials at week 52 and week 24 , respectively $[35,36]$. Similar retention rates have also been observed in patients randomized to receive etanercept treatment after 2 years [37], and the National Swedish registry which assessed the effectiveness of CZP in patients with RA [38].

CZP-treated patients also experienced a rapid improvement in PDUS outcomes, which was then sustained to 1 year. Ultrasound has been shown to have a higher sensitivity and accuracy in detecting inflammation and anatomical damage resulting from RA than clinical and radiographic examinations [39, 40]. Studies have also indicated that PDUS is an effective method of measuring the response of RA symptoms to anti-TNF therapy [41, 42]. This study used PDUS to assess the response of RA (b) ACR20/50/70 responder rate [NRI]

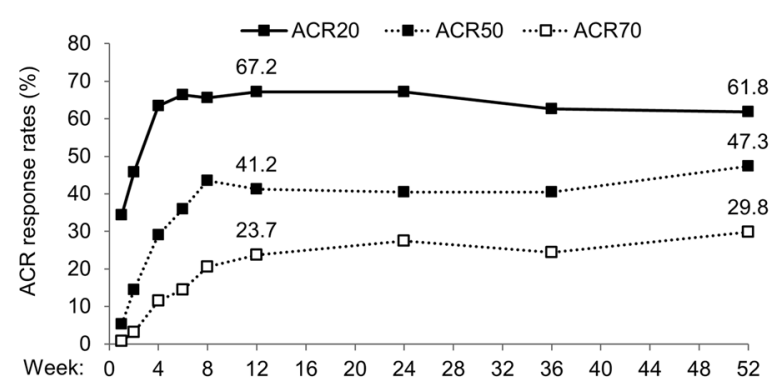

(d) PtAAP [LOCF]

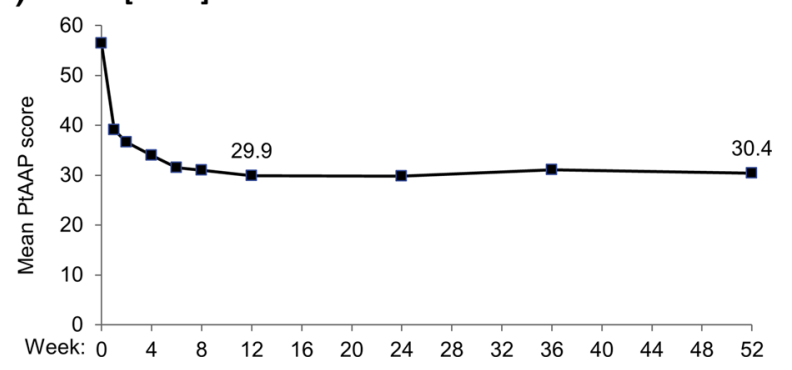

activity score, ESR erythrocyte sedimentation rate, $H A Q-$ $D I$ health assessment questionnaire disability index, $L O C F$ last observation carried forward, NRI non-responder imputation, PtAAP patient assessment of arthritis pain, $V A S$ visual analogue scale $(0-100 \mathrm{~mm})$

patients to CZP treatment and a rapid decrease was observed in joint cavity widening and PD signal/blood flow from the start of CZP treatment up to week 8 , which then remained stable throughout the remainder of the trial period. Mean bone erosion and cartilage damage, measured by PDUS, remained stable throughout the duration of the study. These findings were complemented by stable mean mTSS radiographic measurements to week 52, and PDUS and mTSS measurements were not significantly correlated when compared. Improvement in ultrasound outcomes has been observed in studies investigating other biologic agents, such as adalimumab, etanercept, infliximab, and tocilizumab [43-48] as well as in a recent case study using CZP in an RA patient [49]. Ultrasound bone erosion has also been described as a relapse risk factor in a recent observational study after discontinuation of 

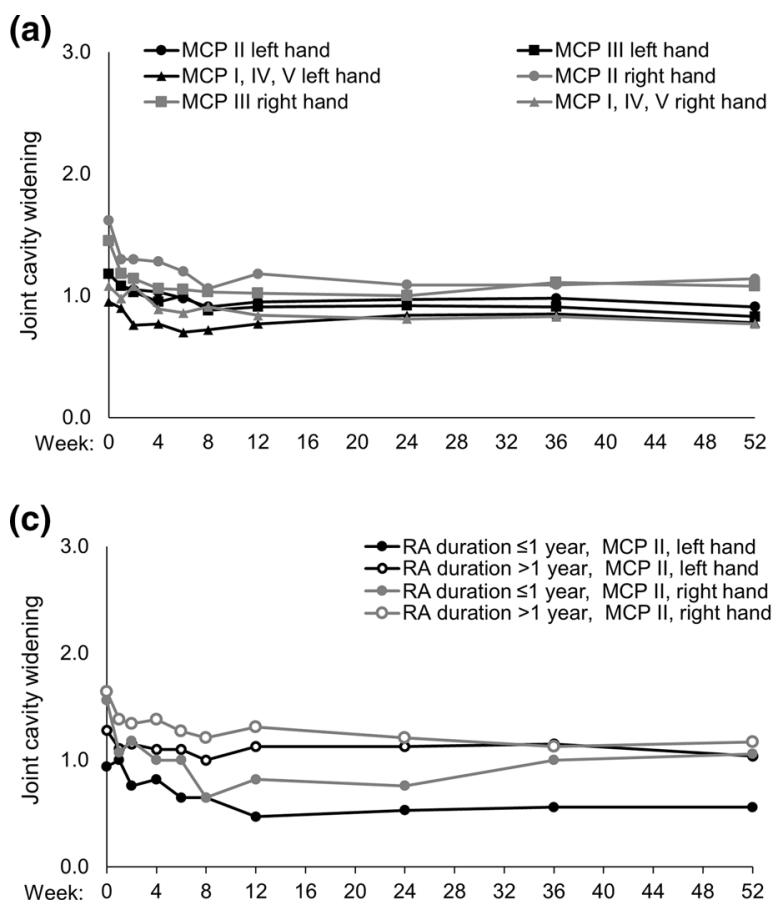

Fig. 6 Mean improvement in joint cavity widening (a, c) and PD signal $(\mathbf{b}, \mathbf{d})$ measured with PDUS over time in metacarpophalangeal joints (MCPs). Groups are analyzed by MCP joint, $n=66$ (a, b) and duration of RA (c, d). Data are reported for the full analysis set in patients eligible for the PDUS subgroup $(n=66)$. Joint cavity widening scores (0-3 scale; $0=$ no effusion/hypertrophy, $1=$ minimal effusion/hypertrophy, $2=$ moderate effusion/hypertrophy, 3 = extensive effusion/hypertrophy). Power Doppler signal was based on the 4-point scale ranging from 0 (normal/minimal blood flow) to 3 (marked

biological therapies [50]. However, because of the heterogeneity of study designs, the studies cannot be directly compared.

RA duration was found to be a predictive baseline characteristic to explain PDUS results over time; post hoc analyses suggest that early RA patients with a disease duration less than 1 year experienced greater reductions in grayscale and PD findings (indicative of inflammation) compared to those with disease duration between 1 and 2 years. However, patient numbers in the subgroup comparisons were low.

In this study, cartilage damage and bone erosion were not found to worsen after the start of treatment and remained stable. This result was consistent with our hypothesis and almost
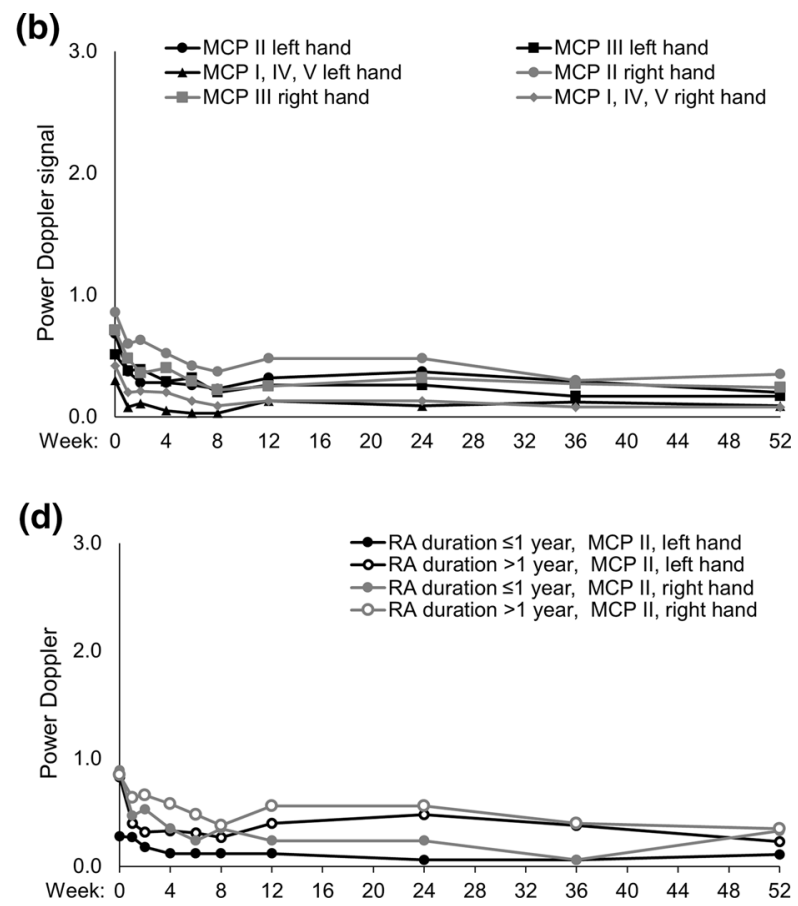

blood flow). Missing values were imputed using last observation carried forward (LOCF). Patients within the PDUS subset had at most 2 years duration of RA and proof of at least two of the following: synovial proliferation, synovial vascularization, or presence of fluid in at least one MCP joint. MCP metacarpophalangeal joints, PD Power Doppler, PDUS Power Doppler ultrasound, $R A$ rheumatoid arthritis

all previous studies that demonstrated that antiTNF treatment alone could slow down the decrease of bone mineral density [51] and cartilage damage, both as monotherapy [52], and when used alongside concomitant DMARD treatment [53]. No new safety signals were identified during this study, comparative with the current knowledge about CZP [54].

Limitations of this study included the openlabel, single-arm study design, which may have introduced bias in favor of treatment response. This study also had a small sample size compared to the RAPID-1 trial [7]; however, our observations support the finding that DAS28(ESR) response rates at week 12 are predictive of disease activity at 52 weeks. Another 
Table 2 Mixed effect repeated measures analysis reporting the overall mean differences in the sum score for joint cavity widening, synovial proliferation, and PD signal and cartilage damage, and bone erosions

\begin{tabular}{|c|c|c|c|c|c|c|}
\hline \multirow[t]{2}{*}{ Fixed effect } & \multicolumn{3}{|c|}{$\begin{array}{l}\text { Sum score for joint cavity widening, } \\
\text { synovial proliferation, and PD signal }\end{array}$} & \multicolumn{3}{|c|}{$\begin{array}{l}\text { Sum score for cartilage damage and } \\
\text { bone erosions }\end{array}$} \\
\hline & $\begin{array}{l}\text { Mean } \\
\text { difference }\end{array}$ & $95 \% \mathrm{CI}$ & $p$ value & $\begin{array}{l}\text { Mean } \\
\text { difference }\end{array}$ & 95\% CI & $p$ value \\
\hline DAS28(ESR) $(\geq 5.1$ vs $<5.1)$ & 1.01 & $\begin{array}{c}-1.61 \\
3.64\end{array}$ & 0.44 & 0.08 & $\begin{array}{c}-4.72 \\
4.89\end{array}$ & 0.97 \\
\hline Gender (female vs male) & 2.27 & $\begin{array}{c}-0.45 \\
4.99\end{array}$ & 0.10 & 1.26 & $\begin{array}{c}-3.71 \\
6.24\end{array}$ & 0.61 \\
\hline Age $(\geq 65$ years vs $<65$ years $)$ & -1.60 & $\begin{array}{c}-4.13 \\
0.93\end{array}$ & 0.21 & 0.67 & $\begin{array}{c}-3.95 \\
5.30\end{array}$ & 0.77 \\
\hline $\begin{array}{l}\text { Duration of } \mathrm{RA}(\geq 1-<2 \text { years vs } \\
\quad<1 \text { year })\end{array}$ & 2.96 & $0.62,5.30$ & 0.01 & 5.31 & $1.03,9.58$ & 0.02 \\
\hline
\end{tabular}

Data are reported for the full analysis set (FAS). Missing data were imputed using last observation carried forward (LOCF). The regression coefficients, 95\% confidence intervals and $p$ values are from a mixed effects linear model for repeated measures with baseline DAS28-ESR score, gender, age, and duration of RA as fixed effects, and week as the repeated measure. PDUS outcomes were analyzed by disease activity (sum score for joint cavity widening, synovial proliferation, and PD signal) and disease severity (sum score for cartilage damage and bone erosions) post hoc. Differences show the overall mean differences between the levels of the independent variables [i.e., DAS28(ESR) $\geq 5.1$ compared to DAS28(ESR) $<$ 5.1]. Mean differences $>0$ indicate that the sum score for the first category of the fixed effect is greater than the second category

$C I$ confidence interval, $D A S$ disease activity score, ESR erythrocyte sedimentation rate, FAS full analysis set, $L O C F$ last observation carried forward, PD Power Doppler, PDUS Power Doppler ultrasound, $R A$ rheumatoid arthritis, SE standard error

limitation was the potential variability in the interpretation of PDUS images, since ultrasonographers were based at individual sites as a result of the multicenter nature of the study. However, this was mitigated by all ultrasonographers being experts who used appropriate equipment and followed predefined recommendations to align the definition of scanning method and standardize scoring. Inter-reader reliability was also quantified through the reassessment of all images by a second experienced ultrasonographer and showed a decrease in reliability over the course of the study. This may be due to the observed decrease in disease activity over time, as variability has been shown to be at a maximum when image subscores are between 1 and 2, where loss of sharpness, or a partial thickness defect, is observed in the cartilage layer [29].

\section{CONCLUSIONS}

This study found that clinical response to week 12 in RA patients treated with CZP may be predictive of response at week 52. A rapid decrease in PDUS-measured joint cavity widening and PD signal was observed to week 8, which was then maintained to week 52 . In addition, no progression in cartilage damage or bone erosion was observed. No new safety signals were identified during this study, comparative with the current knowledge about CZP [54] and other anti-TNF therapies [55].

\section{ACKNOWLEDGEMENTS}

The authors thank the patients, the investigators and their teams who took part in this study. 
Funding. UCB sponsored the study and the development of the manuscript and reviewed the text to ensure that from UCB perspective, the data presented in the publication are scientifically, technically and medically supportable, that they do not contain any information that has the potential to damage the intellectual property of UCB, and that the publication complies with applicable laws, regulations, guidelines and good industry practice. Article processing charges were funded by UCB Pharma. The authors approved the final version to be published after critically revising the manuscript for important intellectual content. All authors had full access to all of the data in this study and take complete responsibility for the integrity of the data and accuracy of the data analysis.

Medical Writing and/or Editorial Assistance Authorship. The authors also acknowledge Susanne Wiegratz, UCB Pharma GmbH, Monheim, Germany for publication coordination, Simone E. Auteri, UCB Pharma, Brussels, Belgium for critical review of the manuscript, and Sarah Jayne Clements, PhD and Maria Haughton, CMPP at Costello Medical, Cambridge, UK, for medical writing and editorial assistance in preparing this manuscript for publication, based on the authors' input and direction. Support for third-party medical writing assistance and article processing changes were funded by UCB Pharma.

Authorship. All named authors meet the International Committee of Medical Journal Editors (ICMJE) criteria for authorship for this article, take responsibility for the integrity of the work as a whole, and have given their approval for this version to be published.

Author Contributions. Substantial contributions to study conception and design: WG; substantial contributions to acquisition of data: EF, ODL, WG; substantial contributions to analysis and interpretation of data: PSP, EF, PLM, AB, LI, ODL, PT, TK, WG; drafting the article or revising it critically for important intellectual content: PSP, EF, PLM, AB, LI, ODL, PT, TK, WG; final approval of the version of the article to be published: PSP, EF, PLM, AB, LI, ODL, PT, TK, WG.

Disclosures. Piercarlo Sarzi-Puttini: Speaker fees; Emilio Filippucci: Speaker fees: AbbVie, BMS, Celgene, Roche, UCB Pharma; Luca Idolazzi: Speaker fees from Celgene, AbbVie, UCB Pharma, and Janssen; Pablo Talavera: Employee of UCB Pharma; Thomas Kumke: Employee of UCB Pharma; Walter Grassi: Consultancies, speaker fees and/or honoraria: AbbVie, BMS, Gruenthal Menarini, MSD, Novartis, Pfizer, and UCB Pharma. Pier Luigi Meroni, Alberto Batticciotto, Orazio De Lucia and Silvano Adami have nothing to disclose.

Compliance with Ethics Guidelines. All procedures performed in studies involving human participants were in accordance with the ethical standards of the responsible Italian authorities [the Agenzia Italiana del Farmaco (AIFA)] and with the 1964 Helsinki declaration and its later amendments or comparable ethical standards. Informed consent was obtained from all individual participants included in the study.

Data Availability. The datasets generated and analyzed during this study are available in anonymized format upon reasonable request via the CSDR platform (http://www. clinicalstudydatarequest.com).

Open Access. This article is distributed under the terms of the Creative Commons Attribution-NonCommercial 4.0 International License (http://creativecommons.org/licenses/ by-nc/4.0/), which permits any noncommercial use, distribution, and reproduction in any medium, provided you give appropriate credit to the original author(s) and the source, provide a link to the Creative Commons license, and indicate if changes were made.

\section{REFERENCES}

1. Smolen JS, Landewé R, Bijlsma J, et al. EULAR recommendations for the management of rheumatoid arthritis with synthetic and biological disease- 
modifying antirheumatic drugs: 2016 update. Ann Rheum Dis. 2017;76(6):960-77.

2. McDonnell T, Ioannou Y, Rahman A. PEGylated drugs in rheumatology-why develop them and do they work? Rheumatology (Oxford). 2014;53(3):391-6.

3. Pasut G. Pegylation of biological molecules and potential benefits: pharmacological properties of certolizumab pegol. BioDrugs. 2014;28(1):15-23.

4. Vashisht P, O'Dell J. Not all TNF inhibitors in rheumatoid arthritis are created equal: important clinical differences. Expert Opin Biol Ther. 2017;17(8):989-99.

5. Fechtenbaum M, Yusof MYM, Emery P. Certolizumab pegol in rheumatoid arthritis: current update. Expert Opin Biol Ther. 2014;14(6):841-50.

6. Palframan R, Airey M, Moore A, Vugler A, Nesbitt A. Use of biofluorescence imaging to compare the distribution of certolizumab pegol, adalimumab, and infliximab in the inflamed paws of mice with collagen-induced arthritis. J Immunol Methods. 2009;348(1):36-41.

7. Van Der Heijde D, Keystone EC, Curtis JR, et al. Timing and magnitude of initial change in disease activity score 28 predicts the likelihood of achieving low disease activity at 1 year in rheumatoid arthritis patients treated with certolizumab pegol: a post hoc analysis of the RAPID 1 trial. J Rheumatol. 2012;39(7):1326-33.

8. Keystone EC, Curtis JR, Fleischmann RM, et al. Rapid improvement in the signs and symptoms of rheumatoid arthritis following certolizumab pegol treatment predicts better longterm outcomes: posthoc analysis of a randomized controlled trial. J Rheumatol. 2011;38(6):990-6.

9. Smolen JS, Breedveld FC, Burmester GR, et al. Treating rheumatoid arthritis to target: 2014 update of the recommendations of an international task force. Ann Rheum Dis. 2016;75(1):3-15.

10. Bhasin S, Cheung PP. The role of power Doppler ultrasonography as disease activity marker in rheumatoid arthritis. Dis Mark. 2015;2015:9.

11. Colebatch AN, Edwards CJ, Østergaard M, et al. EULAR recommendations for the use of imaging of the joints in the clinical management of rheumatoid arthritis. Ann Rheum Dis. 2013;72(6):804-14.

12. Døhn UM, Ejbjerg BJ, Hasselquist M, et al. Are bone erosions detected by magnetic resonance imaging and ultrasonography true erosions? A comparison with computed tomography in rheumatoid arthritis metacarpophalangeal joints. Arthritis Res Ther. 2006;8(4):R110.

13. Filippucci E, Di Geso L, Grassi W. Progress in imaging in rheumatology. Nat Rev Rheumatol. 2014;10(10):628-34.

14. Tămaş M-M, Filippucci E, Becciolini A, et al. Bone erosions in rheumatoid arthritis: ultrasound findings in the early stage of the disease. Rheumatology. 2014;53(6):1100-7.

15. Vreju F, Filippucci E, Gutierrez M, et al. Subclinical ultrasound synovitis in a particular joint is associated with ultrasound evidence of bone erosions in that same joint in rheumatoid patients in clinical remission. Clin Exp Rheumatol. 2016;34(4):673-8.

16. Naredo E, Collado P, Cruz A, et al. Longitudinal power Doppler ultrasonographic assessment of joint inflammatory activity in early rheumatoid arthritis: predictive value in disease activity and radiologic progression. Arthritis Rheum. 2007;57(1):116-24.

17. Sreerangaiah D, Grayer M, Fisher BA, Ho M, Abraham S, Taylor PC. Quantitative power Doppler ultrasound measures of peripheral joint synovitis in poor prognosis early rheumatoid arthritis predict radiographic progression. Rheumatology (Oxford). 2016;55(1):89-93.

18. Ten Cate DF, Luime JJ, Swen N, et al. Role of ultrasonography in diagnosing early rheumatoid arthritis and remission of rheumatoid arthritis-a systematic review of the literature. Arthritis Res Ther. 2013;15(1):R4.

19. Caporali R, Conti F, Alivernini S, et al. Recommendations for the use of biologic therapy in rheumatoid arthritis: update from the Italian Society for Rheumatology I. Efficacy. Clin Exp Rheumatol. 2011;29(3 Suppl 66):S7-14.

20. UCB Pharma. Cimzia ${ }^{\circledR}$ summary of product characteristics: UCB Pharma; 2018. http://www.ema. europa.eu/docs/en_GB/document_library/EPAR_Product_Information/human/001037/WC5000697 63.pdf. Accessed 31 May 2018.

21. Felson DT, Anderson JJ, Boers M, et al. American College of Rheumatology preliminary definition of improvement in rheumatoid arthritis. Arthritis Rheum. 1995;38(6):727-35.

22. van Gestel AM, Haagsma CJ, van Riel PL. Validation of rheumatoid arthritis improvement criteria that include simplified joint counts. Arthritis Rheumatol. 1998;41(10):1845-50. 
23. van der Heijde D. How to read radiographs according to the Sharp/van der Heijde method. J Rheumatol. 2000;27(1):261-3.

24. Sokka T. Radiographic scoring in rheumatoid arthritis. Bull NYU Hosp Jt Dis. 2008;66(2):166-8.

25. Scheel AK, Hermann KG, Kahler E, et al. A novel ultrasonographic synovitis scoring system suitable for analyzing finger joint inflammation in rheumatoid arthritis. Arthritis Rheum. 2005;52(3):733-43.

26. Wakefield RJ, Balint PV, Szkudlarek M, et al. Musculoskeletal ultrasound including definitions for ultrasonographic pathology. J Rheumatol. 2005;32(12):2485-7.

27. Brown AK, Quinn MA, Karim Z, et al. Presence of significant synovitis in rheumatoid arthritis patients with disease-modifying antirheumatic drug-induced clinical remission: evidence from an imaging study may explain structural progression. Arthritis Rheum. 2006;54(12):3761-73.

28. Newman JS, Laing TJ, McCarthy CJ, Adler RS. Power Doppler sonography of synovitis: assessment of therapeutic response-preliminary observations. Radiology. 1996;198(2):582-4.

29. Filippucci E, da Luz KR, Di Geso L, et al. Interobserver reliability of ultrasonography in the assessment of cartilage damage in rheumatoid arthritis. Ann Rheum Dis. 2010;69(10):1845-8.

30. Disler DG, Raymond E, May DA, Wayne JS, McCauley TR. Articular cartilage defects: in vitro evaluation of accuracy and interobserver reliability for detection and grading with US. Radiology. 2000;215(3):846-51.

31. Gutierrez M, Filippucci E, Ruta S, et al. Inter-observer reliability of high-resolution ultrasonography in the assessment of bone erosions in patients with rheumatoid arthritis: experience of an intensive dedicated training programme. Rheumatology (Oxford). 2011;50(2):373-80.

32. Curtis JR, Churchill M, Kivitz A, et al. A randomized trial comparing disease activity measures for the assessment and prediction of response in rheumatoid arthritis patients initiating certolizumab pegol. Arthritis Rheumatol. 2015;67(12):3104-12.

33. Weinblatt ME, Fleischmann $\mathrm{R}$, van Vollenhoven $\mathrm{RF}$, et al. Twenty-eight-week results from the REALISTIC phase IIIb randomized trial: efficacy, safety and predictability of response to certolizumab pegol in a diverse rheumatoid arthritis population. Arthritis Res Ther. 2015;17:325.
34. Mohammed RH, Farahat F, Kewan HH, Bukhari MA. Predictors of European League Against Rheumatism (EULAR) good response, DAS-28 remission and sustained responses to TNF-inhibitors in rheumatoid arthritis: a prospective study in refractory disease. SpringerPlus. 2015;4:207.

35. Strand V, Mease P, Burmester GR, et al. Rapid and sustained improvements in health-related quality of life, fatigue, and other patient-reported outcomes in rheumatoid arthritis patients treated with certolizumab pegol plus methotrexate over 1 year: results from the RAPID 1 randomized controlled trial. Arthritis Res Ther. 2009;11(6):R170.

36. Smolen J, Landewé RB, Mease P, et al. Efficacy and safety of certolizumab pegol plus methotrexate in active rheumatoid arthritis: the RAPID 2 study. A randomised controlled trial. Ann Rheum Dis. 2009;68(6):797-804.

37. Genovese MC, Bathon JM, Martin RW, et al. Etanercept versus methotrexate in patients with early rheumatoid arthritis: two-year radiographic and clinical outcomes. Arthritis Rheum. 2002;46(6):1443-50.

38. Chatzidionysiou K, Kristensen LE, Eriksson J, van Askling J, Vollenhoven R, ARTIS Group. Effectiveness and survival-on-drug of certolizumab pegol in rheumatoid arthritis in clinical practice: results from the national Swedish register. Scand J Rheumatol. 2015;44(6):431-7.

39. Szkudlarek M, Klarlund M, Narvestad E, et al. Ultrasonography of the metacarpophalangeal and proximal interphalangeal joints in rheumatoid arthritis: a comparison with magnetic resonance imaging, conventional radiography and clinical examination. Arthritis Res Ther. 2006;8(2):R52.

40. Walther M, Harms H, Krenn V, Radke S, Faehndrich TP, Gohlke F. Correlation of power Doppler sonography with vascularity of the synovial tissue of the knee joint in patients with osteoarthritis and rheumatoid arthritis. Arthritis Rheum. 2001;44(2):331-8.

41. Naredo E, Moller I, Cruz A, Carmona L, Garrido J. Power Doppler ultrasonographic monitoring of response to anti-tumor necrosis factor therapy in patients with rheumatoid arthritis. Arthritis Rheum. 2008;58(8):2248-56.

42. Seymour MW, Kelly S, Beals CR, et al. Ultrasound of metacarpophalangeal joints is a sensitive and reliable endpoint for drug therapies in rheumatoid arthritis: results of a randomized, two-center placebo-controlled study. Arthritis Res Ther. 2012;14(5):R198. 
43. Iagnocco A, Filippucci E, Perella C, et al. Clinical and ultrasonographic monitoring of response to adalimumab treatment in rheumatoid arthritis. J Rheumatol. 2008;35(1):35-40.

44. Iagnocco A, Perella C, Naredo E, et al. Etanercept in the treatment of rheumatoid arthritis: clinical follow-up over one year by ultrasonography. Clin Rheumatol. 2008;27(4):491-6.

45. Filippucci E, Iagnocco A, Salaffi F, Cerioni A, Valesini G, Grassi W. Power Doppler sonography monitoring of synovial perfusion at the wrist joints in patients with rheumatoid arthritis treated with adalimumab. Ann Rheum Dis. 2006;65(11):1433-7.

46. Taylor PC, Steuer A, Gruber J, et al. Comparison of ultrasonographic assessment of synovitis and joint vascularity with radiographic evaluation in a randomized, placebo-controlled study of infliximab therapy in early rheumatoid arthritis. Arthritis Rheum. 2004;50(4):1107-16.

47. Fiocco U, Ferro F, Vezzu M, et al. Rheumatoid and psoriatic knee synovitis: clinical, grey scale, and power Doppler ultrasound assessment of the response to etanercept. Ann Rheum Dis. 2005;64(6):899-905.

48. Epis O, Filippucci E, Delle Sedie A, De Matthaeis A, Bruschi E. Clinical and ultrasound evaluation of the response to tocilizumab treatment in patients with rheumatoid arthritis: a case series. Rheumatol Int. 2014;34(5):737-42.

49. Kawashiri S-Y, Michitsuji T, Kawakami A. Certolizumab pegol was effective for treating residual synovitis after total knee arthroplasty in a patient with rheumatoid arthritis: therapeutic monitoring by ultrasound. J Med Ultrason. 2018;45(2):371-4.

50. S-y Kawashiri, Fujikawa K, Nishino A, et al. Ultrasound-detected bone erosion is a relapse risk factor after discontinuation of biologic disease-modifying antirheumatic drugs in patients with rheumatoid arthritis whose ultrasound power Doppler synovitis activity and clinical disease activity are well controlled. Arthritis Res Ther. 2017;19(1):108.

51. Manara M, Sinigaglia L. Bone and TNF in rheumatoid arthritis: clinical implications. RMD Open. 2015;1(Suppl 1):e000065.

52. den Broeder AA, Joosten LAB, Saxne T, et al. Long term anti-tumour necrosis factor $\alpha$ monotherapy in rheumatoid arthritis: effect on radiological course and prognostic value of markers of cartilage turnover and endothelial activation. Ann Rheum Dis. 2002;61(4):311-8.

53. Finckh A, Simard JF, Duryea J, et al. The effectiveness of anti-tumor necrosis factor therapy in preventing progressive radiographic joint damage in rheumatoid arthritis: a population-based study. Arthritis Rheum. 2006;54(1):54-9.

54. Bykerk VP, Cush J, Winthrop K, et al. Update on the safety profile of certolizumab pegol in rheumatoid arthritis: an integrated analysis from clinical trials. Ann Rheum Dis. 2015;74(1):96-103.

55. Ramiro S, Sepriano A, Chatzidionysiou K, et al. Safety of synthetic and biological DMARDs: a systematic literature review informing the 2016 update of the EULAR recommendations for management of rheumatoid arthritis. Ann Rheum Dis. 2017;76(6):1101-36. 